\title{
Nanoparticle-modified chitosan-agarose-gelatin scaffold for sustained release of SDF-I and BMP-2
}

This article was published in the following Dove Press journal:

International Journal of Nanomedicine

\author{
Bin Wang' \\ Yuanwei Guo² \\ Xiaofeng Chen ${ }^{3}$ \\ Chao Zeng' \\ Qikang $\mathrm{Hu}^{\prime}$ \\ Wei Yin' \\ Wei Li' \\ Hui Xie' \\ Bingyu Zhang' \\ Xingchun Huang' \\ Fenglei $\mathrm{Yu}^{\prime}$ \\ 'Department of Thoracic Surgery, The \\ Second Xiangya Hospital of Central \\ South University, Changsha 4I00II, \\ People's Republic of China; ${ }^{2}$ Center \\ for Clinical Pathology, Affiliated to The \\ First People's Hospital of Chenzhou, \\ University of South China, Chenzhou \\ 432000, People's Republic of China; \\ ${ }^{3}$ Department of Anesthesiology, The \\ Second Xiangya Hospital of Central \\ South University, Changsha 4I00II, \\ People's Republic of China
}

Correspondence: Fenglei Yu Department of Thoracic Surgery, The Second Xiangya Hospital of Central South University, No. 139 Renmin Road, Changsha, Hunan Province, 4I00II, People's Republic of China

Tel +86 I37 55 I 2999

Fax +86 0731 85295104

Email yufenglei@csu.edu.cn
Background: Stromal cell-derived factor 1 (SDF-1) is an important chemokine for stem cell mobilization, and plays a critical role in mobilization of mesenchymal stem cells (MSCs). Bone morphogenetic protein 2 (BMP-2) plays a critical role in osteogenesis of MSCs. However, the use of SDF-1 and BMP-2 in bone tissue engineering is limited by their short half-lives and rapid degradation in vitro and in vivo.

Methods: The chitosan oligosaccharide/ heparin nanoparticles (CSO/H NPs) were first prepared via self-assembly. Chitosan-agarose-gelatin $(\mathrm{CAG})$ Scaffolds were then synthesized via gelation technology using cross-linked chitosan, agarose, and gelatin, and were modified by CSO/H NPs. The encapsulation efficiency and release kinetics of SDF-1 and BMP-2 were quantified using an enzyme-linked immunosorbent assay. A CCK-8 assays were used to evaluate biocompatibility of NP-modified scaffolds. The biological activity of the loaded SDF-1 and BMP-2 was evaluated using the transwell migration assay and osteogenic induction assay. An animal MSC recruitment model was used to study the ability of SDF-1 released from NP-modified scaffolds to induce migration of MSCs.

Results: In this study, we developed a novel nanoparticle-modified CAG scaffold for the delivery of SDF-1 and BMP-2. CCK-8 assays demonstrated excellent biocompatibility of NP-modified scaffolds. In addition, we investigated the release of SDF-1 and BMP-2 from NP-modified scaffolds, and evaluated the effect of released SDF-1 on MSC migration. The effect of released BMP-2 on MSC osteogenesis was also examined. In vitro cell migration assays showed that SDF-1 released from NP-modified scaffolds retained its migration activity; osteogenesis studies demonstrated that released BMP-2 exhibited a strong ability to induce differentiation towards osteoblasts. Our in vivo recruitment assays showed continuous chemotactic response of MSCs to SDF-1 released from the NP-modified scaffold.

Conclusion: The simplicity of synthesizing CSO/H NP-modified CAG scaffolds, combined with its high cytokine loading capacity and sustained release effect, renders NP-modified CAG scaffold an attractive candidate for sustained release of SDF-1 and BMP-2 to promote bone repair and regeneration.

Keywords: cytokine delivery system, nanoparticles, chitosan-agarose-gelatin scaffold, stromal cell-derived factor-1, bone morphogenetic protein-2

\section{Introduction}

Bone defects occur in many diseases, including osteogenesis imperfecta, trauma, congenital malformations, and tumor resections. ${ }^{1}$ The repair of critical-sized bone defects remains a challenge for reconstructive surgery and usually requires effective large-sized bone excision with nidus and restoration of the mechanical and biological functions of the bone. ${ }^{2,3}$ Traditional approaches for bone reconstruction include autologous bone transplantation, allografts, and the use of artificial implants. ${ }^{4-7}$ Autologous 
bones serve as the ideal materials for bone repair, but the applications are limited by the donor site morbidity, insufficiency, and low availability of bone grafts. ${ }^{4,8}$ Compared to autografts, allografts are associated with the risk of rejection reaction and infection. ${ }^{6,9}$ The use of artificial implants is limited by their lack of biocompatibility. ${ }^{10,11}$ Bone tissue engineering is currently considered a potential alternative to the conventional bone grafts because tissue-engineered substitutes possess unique advantages, such as abundant supply and better biocompatibility. ${ }^{12,13}$ The key elements for bone tissue engineering are seed cells and the scaffold for cell adhesion, proliferation, and differentiation.

Mesenchymal stem cells (MSCs) are the primary seed cells for bone tissue engineering. In comparison to allogeneic MSC transplantation, mobilization of autologous MSCs could avoid the risks of immunoreactions and infection transmission caused by allotransplantation. ${ }^{14}$ Various cytokines are essential for homing, proliferation, and differentiation of MSCs in the process of tissue repair and regeneration. Stromal cell-derived factor 1 (SDF-1) is the most important chemokine in stem cell mobilization and plays a critical role in mobilization of MSCs. ${ }^{15}$ However, bone tissue engineering involves a series of biological processes, which include mobilization of MSCs, osteogenic differentiation, and synthesis of extracellular matrix. Apart from SDF-1 for MSC mobilization, there is a need for another cytokine for osteogenic differentiation of MSCs. Bone morphogenetic protein-2 (BMP-2) plays a critical role in the osteogenesis of MSCs. ${ }^{16}$ However, the short half-lives and the low local concentrations of the cytokines render their application limited. ${ }^{17,18}$ Therefore, a reliable delivery system to localize cytokines to specific tissues for an extended period of time and maintain their bioactivity could improve the homing, differentiation, and proliferation of MSCs at their target tissue. We have previously demonstrated that self-assembled chitosan oligosaccharide/heparin nanoparticles (CSO/H NPs) have a high loading capacity for multiple cytokines. ${ }^{19}$ Both SDF-1 and BMP-2 possess conserved $\mathrm{N}$ terminal amino acid sequences, which could be the heparin-binding sites, ${ }^{19-21}$ and could bind to the heparin of CSO/H NPs. Therefore, CSO/H NPs can be used as an attractive SDF-1 and BMP-2 delivery system for bone tissue engineering.

The CSO/H NPs with loaded cytokines could meet the needs of both bioactive factors and seed cells. Bone tissue engineering requires the establishment of an ideal cell carriage or scaffold. The golden standard scaffold for bone tissue engineering is three-dimensional, highly porous, has interconnected structures, is composed of degradable and biocompatible biomaterials, and safely facilitates the long-term sustained release of bioactive factors. ${ }^{16,22,23}$ The scaffold in this study should be three-dimensional, highly porous, and have interconnected structures that are composed of degradable and biocompatible biomaterials. Most importantly, this scaffold must be able to be modified by the CSO/H NPs. The chitosan-agarose-gelatin (CAG) scaffold was the ideal cell scaffold for this study. The CAG scaffold is composed of chitosan, agarose, and gelatin in varying proportions and has been demonstrated as a potential scaffold for cartilage tissue engineering because of its mechanical strength and porous structure that allow for cell infiltration and proliferation. ${ }^{24,25}$ The interconnected 3D structures with $85 \%$ porosity make the CAG scaffold an attractive candidate for bone tissue regeneration. ${ }^{24}$ Tan et al reported that the positively charged chitosan/heparin nanoparticles can modify the decellularized scaffold by crosslinking to the negatively charged collagen. ${ }^{26}$ We hypothesized that the positively charged $\mathrm{CSO} / \mathrm{H}$ NPs can be used to modify the CAG scaffold by crosslinking to the negatively charged gelatin. Thus, we can prepare a $\mathrm{CSO} / \mathrm{H}$ NP-modified CAG scaffold for sustained dual delivery of SDF-1 and BMP-2 for bone tissue engineering.

In the present work, CSO/H NP-modified CAG scaffolds were prepared. We demonstrated that these nanoparticlemodified CAG scaffolds could not only allow for MSC attachment, but also cytokine loading, maintaining their bioactivity for a long period of time.

\section{Materials and methods Materials}

More than $90 \%$ of deacetylated CSO (Mw 5,000 Da, Zhejiang, People's Republic of China) and chitosan (Mw 20,000 Da, Zhejiang, People's Republic of China) were purchased from Golden-shell pharmaceutical CO. LTD (Zhejiang, People's Republic of China). Low-molecular-weight heparin sodium (Mw 8,000 Da, from Fucus vesiculosus) was supplied by Sigma Chemical Co. (St Louis, MO, USA). Recombinant Human/Mouse/Rat BMP-2 (355-BM/CF) and ELISA kits for BMP-2 were purchased from R\&D Systems (DBP200, Minneapolis, MN, USA). SDF-1 (460-SD-010/ CF) and ELISA kits for SDF-1 were purchased from R\&D Systems (DY 460). Cell Counting Kit-8 (CCK-8, MedChem Express, Monmouth Junction, NJ, USA) was used for the cell proliferation assay. The alkaline phosphatase activity (ALP) microplate test kit (Nanjing Jiancheng Bioengineering Institute, Nanjing, People's Republic of China) was purchased from Nanjing Jiancheng Bioengineering Institute. The size parameters of nanoparticles were measured by laser diffraction (Mastersizer 3000HS, Malvern Instruments Ltd, Malvern, UK). The morphology of the nanoparticles or scaffold was determined by transmission electron microscopy (JEOL, Tokyo, Japan) and scanning electron microscopy 
Table I The characteristics of CSO/HNPs, including composition, size, polydispersity index, and zeta potential

\begin{tabular}{l|l|l|l|l}
\hline Group & $\begin{array}{l}\text { CSO/heparin } \\
(\mathbf{v} / \mathbf{v})\end{array}$ & $\begin{array}{l}\text { Size } \\
(\mathbf{n m})\end{array}$ & PDI & $\begin{array}{l}\text { Zeta potential } \\
(\mathbf{m V})\end{array}$ \\
\hline $\mathrm{I}$ & $4: 1$ & $100.7 \pm 1.6$ & $0.122 \pm 0.012$ & $33.4 \pm \mathrm{I} .1$ \\
2 & $2: 15$ & $92.4 \pm 0.8$ & $0.152 \pm 0.006$ & $-38.2 \pm 0.8$ \\
\hline
\end{tabular}

Note: CSO concentration: $1.0 \mathrm{mg} \mathrm{mL}^{-1}$, heparin concentration: $0.25 \mathrm{mg} \mathrm{mL}^{-1}, \mathrm{n}=4$. Abbreviations: CSO, chitosan oligosaccharide; $\mathrm{H}$, heparin; NPs, nanoparticles; PDI, polydispersity index.

(Hitachi S-3400N, Tokyo, Japan). The fluorescence on mice was detected by the In vivo Imaging System Spectrum (IVIS Spectrum, PerkinElmer, Wellesley, MA, USA).

\section{Preparation and characterization of $\mathrm{CSO} / \mathrm{H} \mathrm{NPs}$}

Self-assembled CSO/H NPs were prepared by self-assembly, and their composition is shown in Table 1. First, low-molecular weight heparin sodium solution $(0.25 \mathrm{mg} / \mathrm{mL})$ and CSO solution $(1.0 \mathrm{mg} / \mathrm{mL})$ were prepared using deionized water. The CSO solution and heparin solution were then mixed in various volume ratios at room temperature and titrated to $\mathrm{pH} 7.35-7.45$ with $1 \%$ sodium hydroxide under magnetic stirring followed by ultrasonication. ${ }^{19,26}$

The particle size distribution and zeta potential of the $\mathrm{CSO} / \mathrm{H}$ NPs were measured by laser diffraction (Mastersizer 3000HS, Malvern Instruments Ltd). The morphology of the nanoparticles selected for further use was determined by transmission electron microscopy (JEOL) and scanning electron microscopy (Hitachi S-3400N) after metal spraying.

\section{Fabrication and characterization of super microporous CAG scaffolds}

CAG scaffolds were synthesized following a previously reported protocol..$^{24,25}$ Briefly, a polymer solution was prepared by dissolving chitosan $(100 \mathrm{mg})$ in $5 \mathrm{~mL}$ of aqueous acetic acid solution (1\%). After complete dissolution of chitosan, an equal amount of gelatin (100 mg) was added to this solution; polymers were dissolved at room temperature using mechanical stirring. Another polymer solution was prepared by dissolving agarose in boiling water $(5 \mathrm{~mL})$. This polymeric solution was cooled down to a temperature of $50^{\circ} \mathrm{C}-55^{\circ} \mathrm{C}$. Thereafter, both the polymeric solutions were mixed together. Glutaraldehyde $(0.5 \mathrm{~mL} 0.2 \%$ [v/v] was added to this polymeric blend and mixed thoroughly. The solution mixture was poured into precooled syringes and incubated at $-12^{\circ} \mathrm{C}$ for 16 hours, resulting in the formation of an interconnected porous scaffold. The synthesized scaffolds were thawed and washed using deionized water to remove the unreacted crosslinker. Scaffolds were then freeze-dried and stored for further use.

Determination of the porosity, swelling ratio, degree of degradation, and the flow characteristics were in accordance with the method previously reported. ${ }^{24}$ Detailed methods can be found in the reported protocol and are not repeated in this article.

\section{Immobilization of CSO/H NPs onto scaffolds and then loading SDF-I and BMP-2}

To prepare CSO/H NP-modified CAG scaffolds, the CAG scaffolds (diameter: $13 \mathrm{~mm}$ and thickness: $2 \mathrm{~mm}$ ) were cut from the primary samples. The scaffolds were placed in $5 \mathrm{mg} / \mathrm{mL}$ of NP solution for 2 hours and then washed with fresh PBS three times, 5 minutes per wash. Glutaraldehyde $(0.5 \mathrm{~mL} 0.2 \%[\mathrm{v} / \mathrm{v}])$ was added to the nanoparticle-treated scaffolds in $5 \mathrm{~mL}$ deionized water and continuously incubated for 2 hours at $37^{\circ} \mathrm{C}$, followed by washing with deionized water to remove the unreacted crosslinker. Treated scaffolds were then freeze-dried and stored for further use. To evaluate the loading content of $\mathrm{CSO} / \mathrm{H}$ NPs in the CAG scaffolds, the nanoparticle-modified CAG scaffolds and unmodified CAG scaffolds were weighed using an analytical balance (ATY124, Shimadzu, Tokyo, Japan). Modification efficiency or immobilizing content was determined by the following equation: ${ }^{27}$

$$
\begin{aligned}
& \text { NPs immobilizing content } \\
& =\frac{\text { Weight of NPs modified CAG }- \text { Weight of CAG }}{\text { Weight of CAG }}
\end{aligned}
$$

The scaffolds immobilized with CSO/H NPs were imaged by environmental scanning electron microscopy (Hitachi S-3400N, Tokyo, Japan) after metal spraying.

To localize SDF-1 and BMP-2, modified scaffolds were immersed in different concentrations of SDF-1 or BMP-2 solutions for 4 hours at room temperature. To evaluate the encapsulation efficiency of SDF-1 and BMP-2 in the CSO/H NPs, the amounts of the free cytokines in the solution were determined by ELISA. Drug encapsulation efficiency was calculated by the following equation: ${ }^{27}$

\section{Encapsulation efficiency $(\%)=$}

Total amount of cytokines added - Free cytokines

Total amount of cytokines added

\section{In vitro release}

The NP-modified scaffolds with loaded cytokines were placed in $10 \mathrm{~mL}$ tubes in triplicate. The samples contained 
$300 \mathrm{ng}$ of BMP-2 or $300 \mathrm{ng}$ of SDF-1. PBS (3 mL) was then added into each tube and incubated at $37^{\circ} \mathrm{C}$ with $100 \mathrm{rpm}$ agitation. At the preset time points, the PBS in the tubes was collected and replaced. The concentration of cytokines in the PBS was determined by an SDF-1 ELISA kit (DY 460, Minneapolis, MN, USA) and BMP-2 ELISA kit (DBP200, Minneapolis, MN, USA). The cytokine ELISAs were performed according to the manufacturer instructions using a standard curve. The amount of cytokines released was determined from a calibration curve based on known concentrations of SDF-1 or BMP-2.

\section{Preparation of MSCs and labeling with green fluorescent protein}

MSCs were extracted from 2- to 3-week-old C57BL/6 female mice in accordance with the reported protocol. ${ }^{28}$ Briefly, the animals were sacrificed, and the marrow in the humeri, tibias, and femurs was flushed with Minimum Essential Medium/ Earle's Balanced Salt Solution (Thermo Fisher Scientific, Waltham, MA, USA). Subsequently, the bones were cut into $1-3 \mathrm{~mm}^{2}$ bone chips and digested by collagenase II for $1-2$ hours in a shaking incubator at $37^{\circ} \mathrm{C}$ with a shaking speed of $200 \mathrm{rpm}$. Next, the hips were resuspended in normal culture medium containing MEM (Thermo Fisher Scientific) supplemented with 10\% fetal bovine serum (Gibco, Grand Island, NY, USA) and 1\% antibiotic - penicillin and streptomycin solution (Sigma Aldrich) in a $25-\mathrm{cm}^{2}$ plastic culture flask. The MSCs migrated from the bone fragments in the days that followed. The flask was kept in a humidified $5 \% \mathrm{CO}_{2}$ incubator at $37^{\circ} \mathrm{C}$ for 72 hours. The medium was replenished every 3 days up to passages $2-4$.

Green fluorescent protein (GFP)-labeled MSCs were prepared with Lipofectamine ${ }^{\circledR} 3000$ reagent (Thermo Fisher Scientific) according to the instructions. The DNA transfected contained the GFP gene and anti-puromycin gene, and the GFP-labeled MSCs were screened by normal culture medium supplemented with $1 \%$ puromycin.

All procedures in this study involving animals were approved by the animal ethics committee of Central South University, People's Republic of China and we followed the Laboratory animal Guideline for ethical review of animal welfare (GB/T 35892-2018).

\section{In vitro cytotoxicity and cell compatibility model}

The CCK-8 assay was applied to study the cytotoxicity of the NP-modified CAG scaffolds and whether they inhibit proliferation of MSCs. First, $5 \times 10^{3}$ MSCs were seeded in normal culture medium into each well of a $24-w e l l$ plate. The cells were then cultured for different periods of time (1-5 days after cells seeded) with modified scaffolds or unmodified scaffolds. The scaffolds were immersed in the medium but kept away from contact with the cells by a customized Transwell. Following a predetermined period of incubation, we removed the scaffolds and medium. Then, $400 \mu \mathrm{L}$ medium and $40 \mu \mathrm{L}$ of CCK-8 solution were successively added to each well. The cells were then incubated at $37^{\circ} \mathrm{C}$ for 4 hours. The optical density was measured at $490 \mathrm{~nm}$ using a multiscan spectrometer (Varioskan Flash, Thermo Electron Corporation, Wilmington, DE, USA). The optical density is linearly proportional to the number of living cells in the sample.

To observe the cell compatibility of the modified scaffolds, we seeded the MSCs onto the scaffolds and then the scaffolds with MSCs were incubated in a 24 well-plate. The plate was kept in a humidified $5 \% \mathrm{CO}_{2}$ incubator at $37^{\circ} \mathrm{C}$. The cell compatibility of materials was determined by assessing the density of cells attached to the scaffolds and the morphology of attached cells 2 days after cells were seeded.

\section{In vitro model of MSC migration induced by SDF-I released from NP-modified scaffolds}

A Transwell migration model was used to study the ability of SDF-1 released from NP-modified scaffolds to induce migration of MSCs. Migration assays were carried out in a 24-well Transwell using polycarbonate membranes with 8 - $\mu \mathrm{m}$ pores (Corning Costar, Cambridge, MA, USA). Next, MSCs at a density of $4 \times 10^{5}$ cells $/ \mathrm{mL}$ in $100 \mu \mathrm{L}$ of medium were placed in the upper chamber of the Transwell assembly. Following a predetermined period of incubation, the upper surface of the membrane was gently swabbed to remove nonmigrating cells and then washed twice with PBS. The membrane was then fixed in 5\% glutaraldehyde for 30 minutes and stained with $0.5 \%$ crystal violet for 10 minutes at room temperature. The number of migrating cells was determined by counting cells in five randomly selected fields per well under the microscope at a magnification of $100 \times$.

\section{In vitro MSC osteogenesis induced by BMP-2 released from NP-modified scaffolds}

The activity of BMP-2 released from the NP-modified scaffold was detected by an osteogenic induction assay. MSCs $\left(4 \times 10^{4}\right)$ were seeded in each well of a 24-well plate with normal culture medium, and the NP-modified scaffolds with 300 ng BMP-2 loaded were immersed in medium but kept away from contact with the cells. Three groups of culture plates were treated with 
normal culture medium (negative control), normal culture medium supplemented with 300 ng BMP-2, and osteogenesis induction culture medium (positive control), respectively. Following a predetermined period of incubation $(7,14$, and 21 days) in a humidified $5 \% \mathrm{CO}_{2}$ incubator at $37^{\circ} \mathrm{C}$, the ALP in each well was measured using an ALP microplate test kit (Nanjing Jiancheng Bioengineering Institute, Nanjing, People's Republic of China), and the samples in each well were stained with $0.1 \%$ alizarin red and imaged with a microscope.

\section{In vivo model of MSC recruitment induced by SDF-I released from NP-modified scaffolds}

An animal MSC recruitment model was used to study the ability of SDF-1 released from NP-modified scaffolds to induce migration of MSCs. Male athymic nude mice (7 weeks old) received implantation of NP-modified scaffolds with loaded SDF-1 or NP-modified scaffolds alone. The scaffolds were implanted subcutaneously into the back of each mouse after anesthesia with pentobarbital $(50 \mathrm{mg} / \mathrm{kg})$. Following a predetermined period $\left(1,3,7,10,15\right.$, and 18 days), $1 \times 10^{6}$ GFP-MSCs in $100 \mu \mathrm{L}$ was injected into the veins of the tails. The mice were observed 48 hours later by the IVIS Spectrum (PerkinElmer) to detect chemotaxis of SDF-1 released from NP-modified scaffolds after the predetermined time. The chemotaxis of SDF-1 was determined by green fluorescence intensity of the implant position.

\section{Statistical analysis}

The experimental values are expressed as means \pm SD. Two-way ANOVA and Student's $t$-test were performed by using GraphPad Prism (v6.04, GraphPad Software, La Jolla, CA, USA) software. $P<0.05$ indicates statistical significance.

\section{Results}

\section{Characterization of CSO/H NPs}

Based on previous research results, we further optimized the ratio of CSO and heparin, and obtained nanoparticles with better size parameters. Characterization of the CSO/H NPs is shown in Table 1. The average particle sizes in the two groups were 100.7 and $92.4 \mathrm{~nm}$. Nanoparticles in both of the groups had a good polydispersity index (PDI; <0.25). The zeta potentials in the two groups were also favorable (absolute values $>30 \mathrm{mV}$ ). The optimal size of nanoparticles designed for drug delivery is $50-150 \mathrm{~nm}$, to confer a high surface area-to-volume ratio. The relatively low PDI and greater absolute values of zeta potential indicate that the size distribution of the nanoparticles was uniform, and the particles can be more stably suspended, which is suitable for the requirements of this study. We selected the group $1 \mathrm{CSO} / \mathrm{H}$ NPs for further study because the positively charged particles (zeta potential $\sim 33.4 \mathrm{mV}$ ) can modify the CAG scaffold by crosslinking to the negatively charged gelatin. Figure $1 \mathrm{~A}$ shows the size distribution and Figure 1B-F shows the transmission electron microscope images (Figure 1B-D) and scanning electron microscope images (Figure 1E and F) for these $\mathrm{CSO} / \mathrm{HNPs}$, which were spherical with a mean diameter of $\sim 100.7 \mathrm{~nm}$, suggesting an ideal size for drug delivery.

\section{Characterization of CAG scaffolds and scaffolds with immobilized nanoparticles}

Among the different combinations of varying polymer concentrations, we found good mechanical stability when using $1 \%$ chitosan with $2 \%-3 \%$ agarose and $1 \%$ gelatin concentrations in the polymer solution mixture (Table 2). The appearance and the microstructure under SEM of CAG scaffolds are shown in Figure 2A and B. The CAG scaffold exhibited the ideal characteristics of a cell scaffold, such as a three-dimensional structure, high porosity, and high surface area-to-volume ratio. The microporous structure of the CAG scaffolds consisted of interconnected pores with an average diameter of $100 \mu \mathrm{m}$ and a thin pore wall (Figure 2B). As shown in Table 2, both of the CAG scaffolds had a high porosity, swelling ratio, and flow rate, which is suitable for cell adhesion, infiltration, and proliferation.

As shown in Figure 3 and Table 2, both the CAG scaffolds could be modified by $\mathrm{CSO} / \mathrm{H}$ NPs. For immobilizing NPs, the group $1 \mathrm{CAG}$ scaffold could immobilize many more NPs into the pores than the scaffold of group 2. With a relatively high content of gelatin, the group $1 \mathrm{CAG}$ scaffold had more exposed negatively charged radicals, which could be adsorbed by positively charged CSO/H NPs. Therefore, we selected the group $1 \mathrm{CAG}$ scaffold for further analysis because of its advantageous content for immobilizing NPs.

\section{SDF-I and BMP-2 encapsulation efficiency and in vitro release}

Table 3 shows the cytokines encapsulation efficiency of the NP-modified CAG scaffold. The NP-modified CAG scaffold had a high encapsulation efficiency $(>80 \%)$ of both SDF-1 and BMP-2. The physiological concentration of the cytokines is much lower than $100 \mathrm{ng} \mathrm{mL} \mathrm{m}^{-1}$. Therefore, the cytokine loading content is sufficient to meet the needs required by tissue engineering.

The in vitro release profiles of the cytokines (SDF-1 and BMP-2) loaded in the NP-modified scaffolds are shown in Figure 4. The NP-modified scaffolds with loaded SDF-1 displayed a typical initial burst release in the first 3 days and 
A
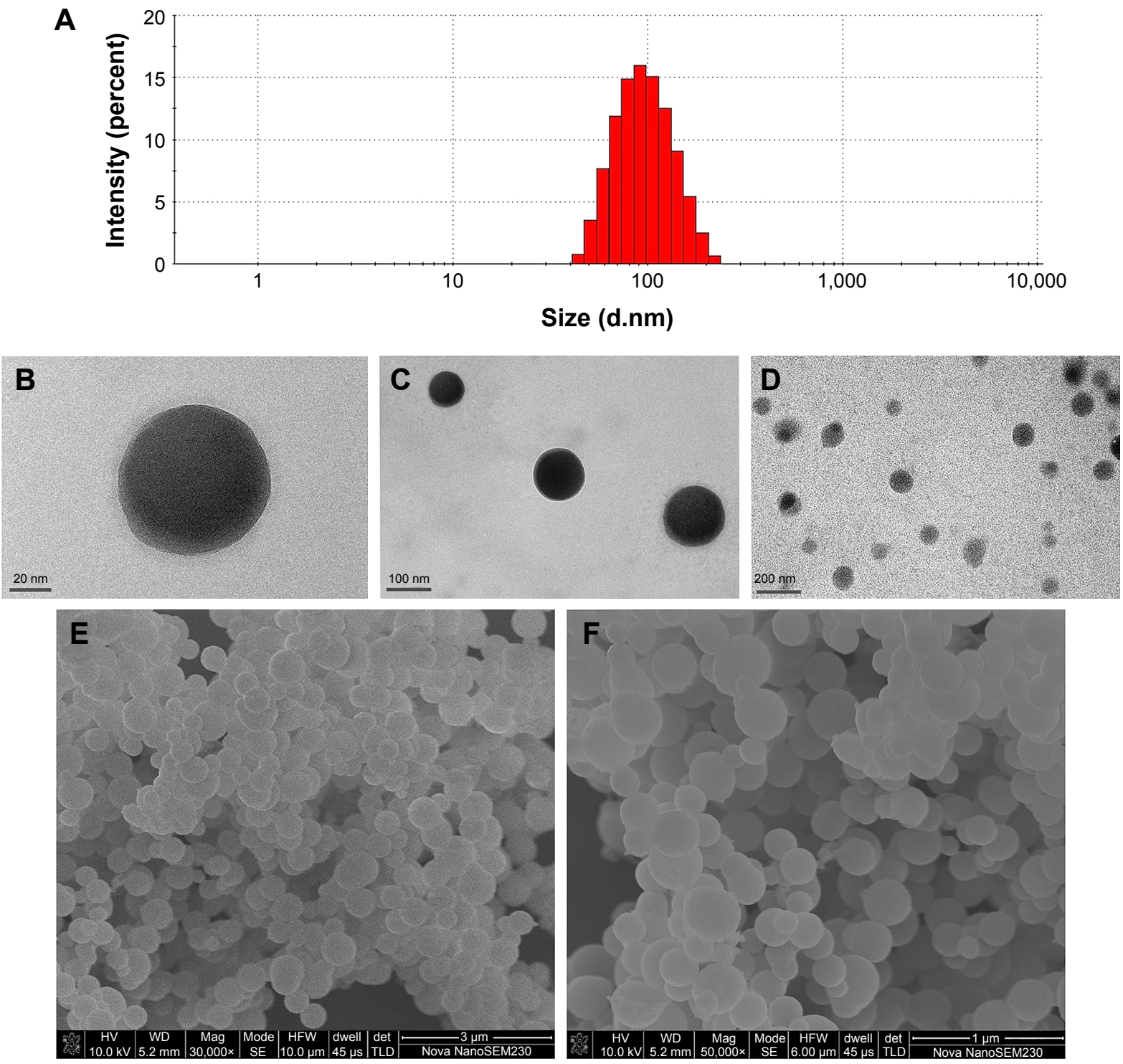

Figure I Characterization of CSO/H NPs.

Notes: (A) Size distribution, (B-D) TEM images, and (E, F) SEM images of group I CSO/H NPs.

Abbreviations: CSO, chitosan oligosaccharide; $\mathrm{H}$, heparin; NPs, nanoparticles; SEM, scanning electron microscopy; TEM, transmission electron microscopy.

Table 2 The characteristics of chitosan-agarose-gelatin cryogels and the immobilized content of $\mathrm{CHO} / \mathrm{H} \mathrm{NPs}$

\begin{tabular}{|c|c|c|}
\hline Group & $\mathrm{I}$ & 2 \\
\hline Chitosan-agarose-gelatin content (\%) & 4 & 5 \\
\hline Chitosan-agarose ratio & $1: 2$ & $1: 3$ \\
\hline Gelatin (\%) & I & I \\
\hline Glutaraldehyde (\%) & 0.2 & 0.2 \\
\hline Porosity (\%) & 82 & 79 \\
\hline Swelling ratio (\%) & 94.7 & 91.2 \\
\hline Flow rate $\left(\mathrm{mL} \mathrm{min}{ }^{-1}\right)$ & Up to 10 & Up to 10 \\
\hline Degree of degradation (\%) & 15.63 & 14.24 \\
\hline Immobilizing content of NPs $\left(\mu \mathrm{g} \mathrm{mg}^{-1}\right)$ & $17.52 \pm 4.16$ & $10.76 \pm 2.28$ \\
\hline
\end{tabular}

Note: The content of chitosan, agarose, or gelatin in this table is the weight added in water.

Abbreviations: CSO, chitosan oligosaccharide; $\mathrm{H}$, heparin; NPs, nanoparticles. exhibited a gentler release pattern after 5 days. In contrast, the release profiles of BMP-2 exhibited a gentler release pattern similar to a zero-order release pattern in kinetics. However, NP-modified scaffolds loaded with both SDF-1 and BMP-2 effectively achieved sustained release profiles for more than 15 days. Sustained release of cytokines could maintain the bioactivity of the cytokines loaded in the NP-modified scaffold for a long period of time.

\section{In vitro cytotoxicity and cell compatibility model}

A CCK-8 assay was used to evaluate the cytotoxic effects of the NP-modified CAG scaffold or unmodified scaffold 

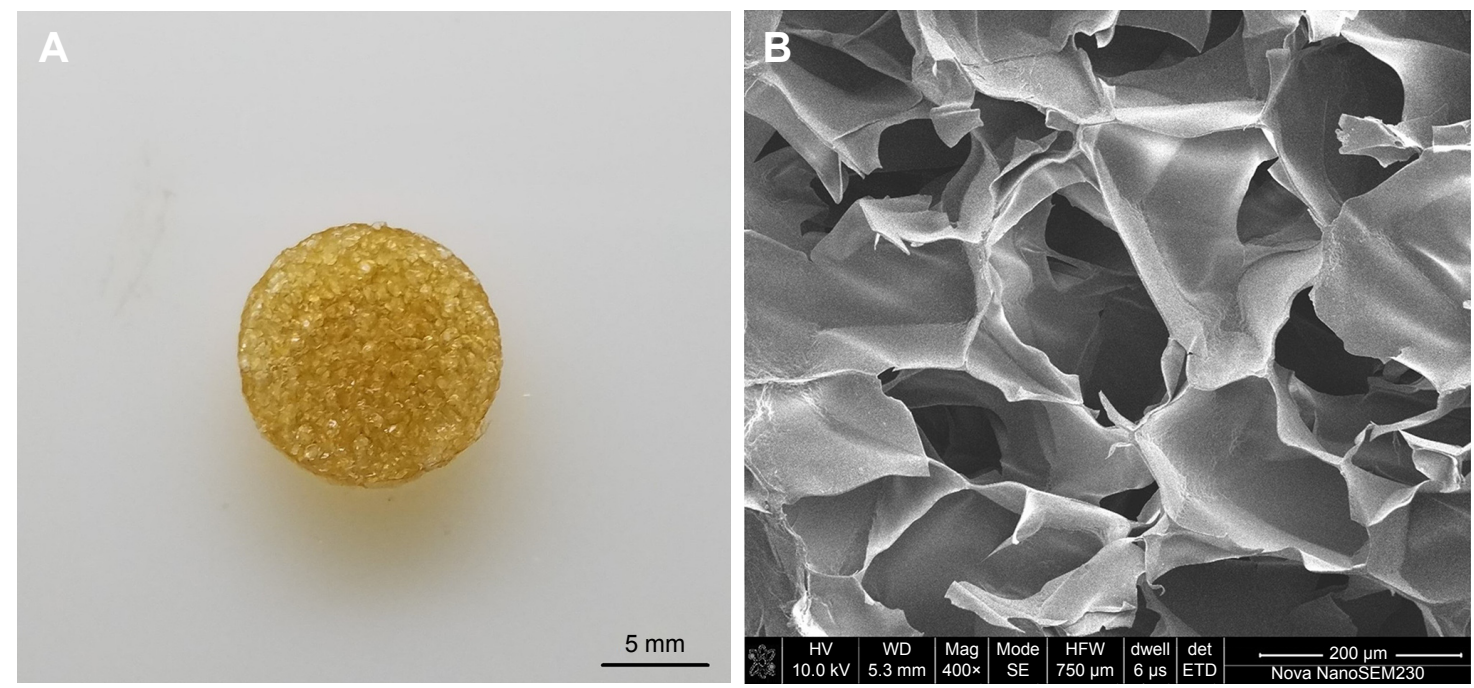

Figure 2 Characterization of CAG scaffold.

Notes: Appearance (A) and SEM images (B) of CAG scaffold.

Abbreviations: CAG, chitosan-agarose-gelatin; SEM, scanning electron microscopy.
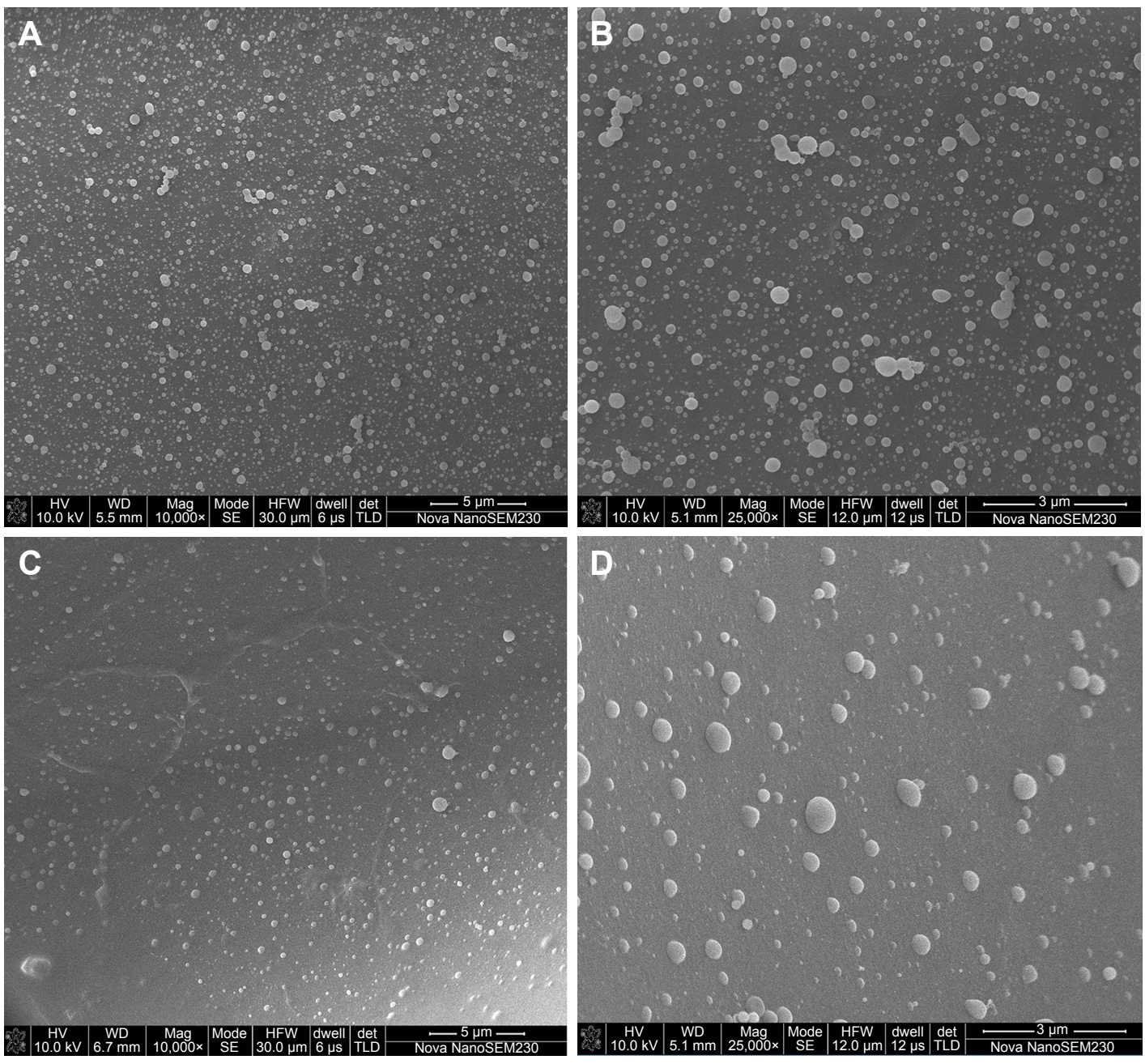

Figure 3 SEM images of CSO/H NP-modified CAG scaffold.

Note: (A, B) Group I scaffold modified with NPs; (C, D) Group 2 scaffold modified with NPs.

Abbreviations: CAG, chitosan-agarose-gelatin; CSO, chitosan oligosaccharide; $\mathrm{H}$, heparin; NP, nanoparticle; SEM, scanning electron microscopy. 
Table 3 The cytokines encapsulation efficiency of CSO/H NPsmodified CAG scaffold in various cytokines/NP (w/w) ratios

\begin{tabular}{l|l|l|l|l}
\hline Group & $\mathbf{2} \boldsymbol{\mu g} / \mathbf{m g}$ & $\mathbf{I} \boldsymbol{\mu g} / \mathbf{m g}$ & $\mathbf{5 0 0} \mathbf{~ g} / \mathbf{m g}$ & $\mathbf{2 5 0} \mathbf{~ g /} / \mathbf{m g}$ \\
\hline SDF-I EE (\%) & $89.54 \pm 1.39$ & $94.37 \pm 1.48$ & $94.41 \pm 1.37$ & $93.53 \pm 1.87$ \\
BMP-2 EE (\%) & $82.73 \pm 2.95$ & $92.67 \pm 1.82$ & $92.85 \pm 1.52$ & $93.78 \pm 1.25$
\end{tabular}

Note: The cytokines/NP (w/w) ratios are $2 \mu \mathrm{g}, 1 \mu \mathrm{g}, 500 \mathrm{ng}$ and $250 \mathrm{ng}$ I mg. Abbreviations: BMP-2, bone morphogenetic protein-2; CAG, chitosan-agarosegelatin; CSO, chitosan oligosaccharide; EE, encapsulation efficiency; H, heparin; NP, nanoparticle; SDF-I, stromal cell-derived factor I.

on MSCs, which were cultured in $400 \mu \mathrm{L}$ of normal culture medium with the scaffolds immersed (Figure 5A). As shown in Figure 5B, the proliferation ability of MSCs among the three groups was not significantly different on days 1 and 2 . There was a significant difference in the proliferation ability between the CAG-NP group and the control group on days $3-5(P<0.05)$. The OD value of the two groups fluctuated during the last 3 days. There was only a significant difference in the OD value between the CAG group and the control group on day 3 , and as a whole, the proliferation ability of MSCs in the two groups was not significantly different. As shown in Figure 5B, the NP-modified CAG scaffold or unmodified scaffold showed no significant cytotoxicity to MSCs. Figure 5C and D shows a high density of MSCs attached to the scaffolds, and the shape of the attached cells was fusiform or polygon, which is indicative of healthy cells. The results demonstrated excellent cell compatibility of the NP-modified CAG scaffolds.

\section{Effect of SDF-I released from NP- modified scaffolds on migration of MSCs}

The ability of released SDF-1 to induce chemotaxis in vitro was tested using a Transwell system (Figure 6A). Migration of MSCs across the transmembrane was then quantified

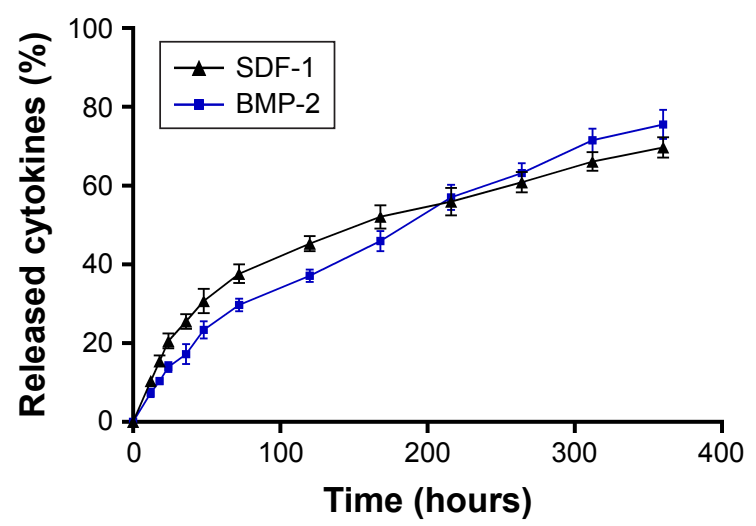

Figure 4 Release kinetics of SDF-I and BMP-2 from the NP-modified CAG scaffold were measured using an ELISA, $n=3$ for each group.

Abbreviations: BMP-2, bone morphogenic protein-2; CAG, chitosan-agarosegelatin; SDF-I, stromal cell-derived factor I. by removing the cells from the seeded side and staining the underside of the membrane with crystal violet. SDF-1 induced migration of the MSCs across the Transwell membrane. MSCs were then induced for 12-72 hours by the NP-modified scaffolds alone, SDF-1 alone, or NP-modified scaffolds with loaded SDF-1. There was no statistically significant difference in the numbers of MSCs crossing the transmembrane between the SDF-1 alone group and SDF$1-N P$-scaffold group until 48 hours of incubation $(P<0.01$, Figure $6 \mathrm{~B}$ and $\mathrm{C}$ ), indicating that the NP-modified scaffolds could maintain the bioactivity of SDF-1 for a long period of time.

\section{Effect of BMP-2 released from NP-modified scaffolds on osteogenesis of MSCs}

Figure 7 presents the results of the analysis of the ALP activity and alizarin red staining of MSCs seeded onto 24-well plates and cultured with the materials for up to 21 days, respectively. As shown in Figure 7A, much lower ALP activity was observed in the groups lacking induction medium (CAG-NPs) than in the other groups, implying that the pure scaffold or the nanoparticles from the scaffold do not improve early osteogenesis (ALP activity). In contrast, the other groups (BMP-2, CAG-NPs loaded with BMP-2, and positive control) showed significantly improved ALP activities. The ALP activity of the cells exposed to CAGNPs loaded with BMP-2 exhibited a similar tendency to that obtained with the BMP-2 group in the first week. However, the ALP activity continued to increase in the BMP-2-loaded groups, exceed the BMP-2 group $(P<0.01)$, and get close to that of the induction media group $(P<0.05)$ over the 14 days. It is possible that the sustained release of BMP-2 led to continuous osteogenesis induction, and the ALP activity in BMP-2-loaded groups eventually exceeded that of the induction media group, but not significantly $(P=0.09)$.

The calcium depositions and calcium nodes were further characterized through alizarin red staining 7, 14, and 21 days after the MSCs were seeded. As shown in Figure 7B, cells in the positive control, the BMP-2, and CAG-NPs with loaded BMP-2 groups exhibited positive staining, whereas the NP-modified CAG scaffold group had almost no positive staining, implying that the scaffold or the nanoparticles have no significant positive effect on the promotion of osteogenic differentiation of MSCs. Compared with the BMP-2 group, the NPs CAG scaffolds with loaded BMP-2 also showed more significant positive staining in the last 2 weeks. More importantly, the CAG-NPs with loaded BMP-2 group presented the most significant positive staining among these 
A

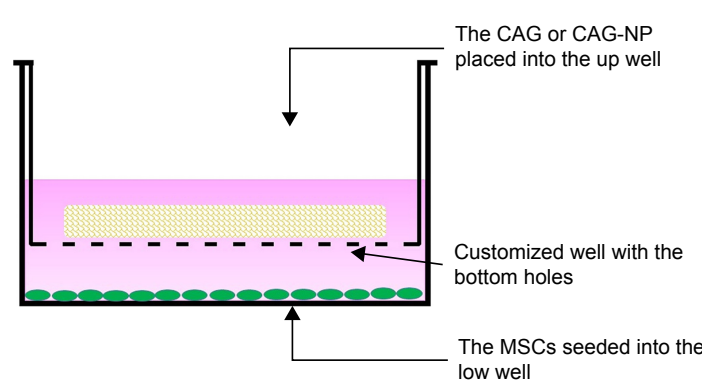

B

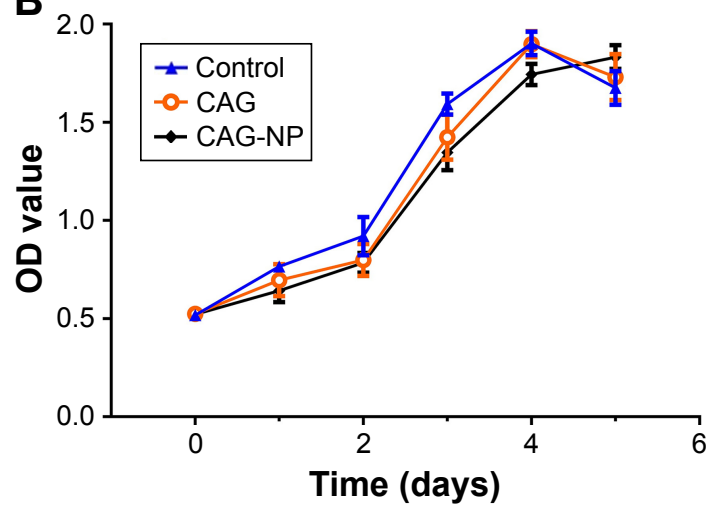

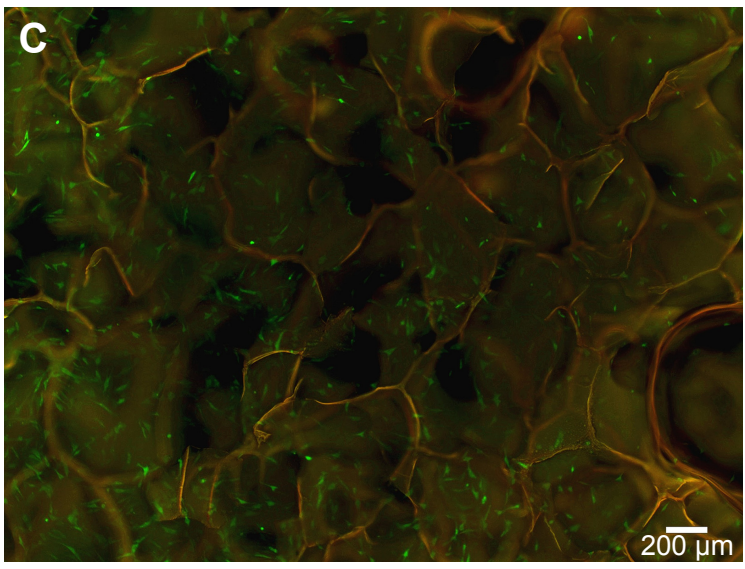

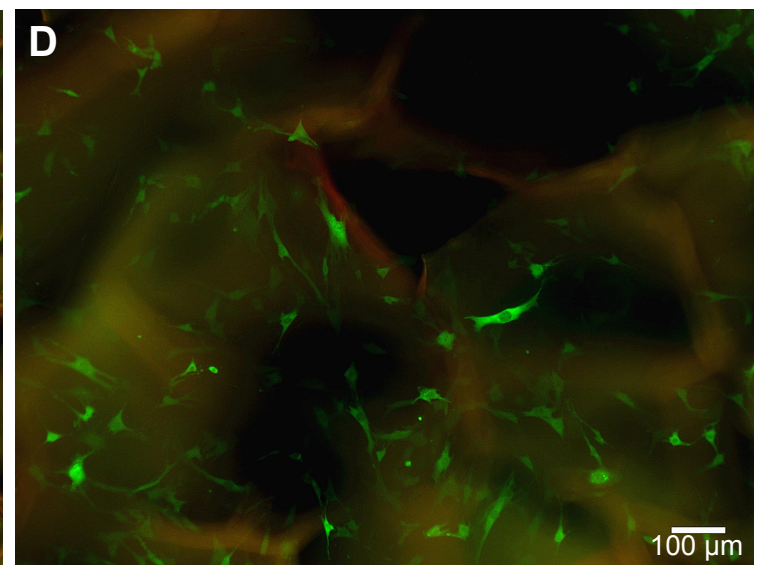

Figure 5 A CCK-8 assay was used to evaluate the cytotoxic effects of the NP-modified CAG scaffold or unmodified scaffold on MSCs.

Notes: (A) Pictorial images of the in vitro cytotoxicity model. (B) Cell proliferation test using the CCK-8 assay. There is a significant difference in proliferation ability between the CAG-NP group and the control group on days 3-5 $(P<0.05)$. The OD value of the two groups fluctuated during the last 3 days. There was only a significant difference in OD value of the CAG group and the control group on day $3(P<0.05)$. (C, D) Representative image of MSCs attached to the NP-modified scaffolds and the morphology of the attached cells, $n=5$ for each group.

Abbreviations: CAG, chitosan-agarose-gelatin; CCK-8, Cell Counting Kit-8; MSCs, mesenchymal stem cells; NP, nanoparticle.

groups after 21 days incubation, implying a synergistic effect of BMP-2 and the delivery system in osteoinduction.

\section{In vivo recruitment of MSCs by SDF-I released from NP-modified scaffolds}

The ability of released SDF-1 to induce chemotaxis in vivo was tested using an animal MSC recruitment model (Figure 8 ). The MSCs recruited to the target position by SDF-1 released from NP-modified scaffolds were detected with the IVIS Spectrum. As Figure 8 shows, a relatively high luminance of fluorescence was excited in the first 10 days. The fluorescence became much weaker on the 15 th day and then vanished eventually. On the contrary, the control group showed no fluorescence at every time point (Figure 8). This result indicates that SDF-1 released from NP-modified scaffolds could recruit MSCs in vivo for more than 2 weeks. The recruitment of MSCs by released SDF-1 may be a better solution for supplying seed cells for bone tissue engineering.

\section{Discussion}

Bone tissue engineering is the most likely alternative to the conventional bone grafts because tissue-engineered substitutes possess unique advantages, such as abundant supply, self-repairing capability, and better biocompatibility. ${ }^{12,13}$ The key elements for bone tissue engineering are seed cells and the scaffold for cell adhesion, proliferation, and differentiation. In this study, we demonstrated that the NPs-modified CAG scaffold with cytokines loaded almost covers these key elements of tissue engineering.

Nanoparticles constructed with heparin have been studied as cytokine delivery carriers for years because many cytokines, such as stromal SDF-1, BMP-2, vascular endothelial growth factor, placental growth factor, platelet-derived growth factor, and fibroblast growth factor, ${ }^{29-33}$ can bind to heparin through their conserved amino acid sequences. The use of chitosan and heparin for the delivery of bioactive molecules has many advantages, including being inexpensive, 
A
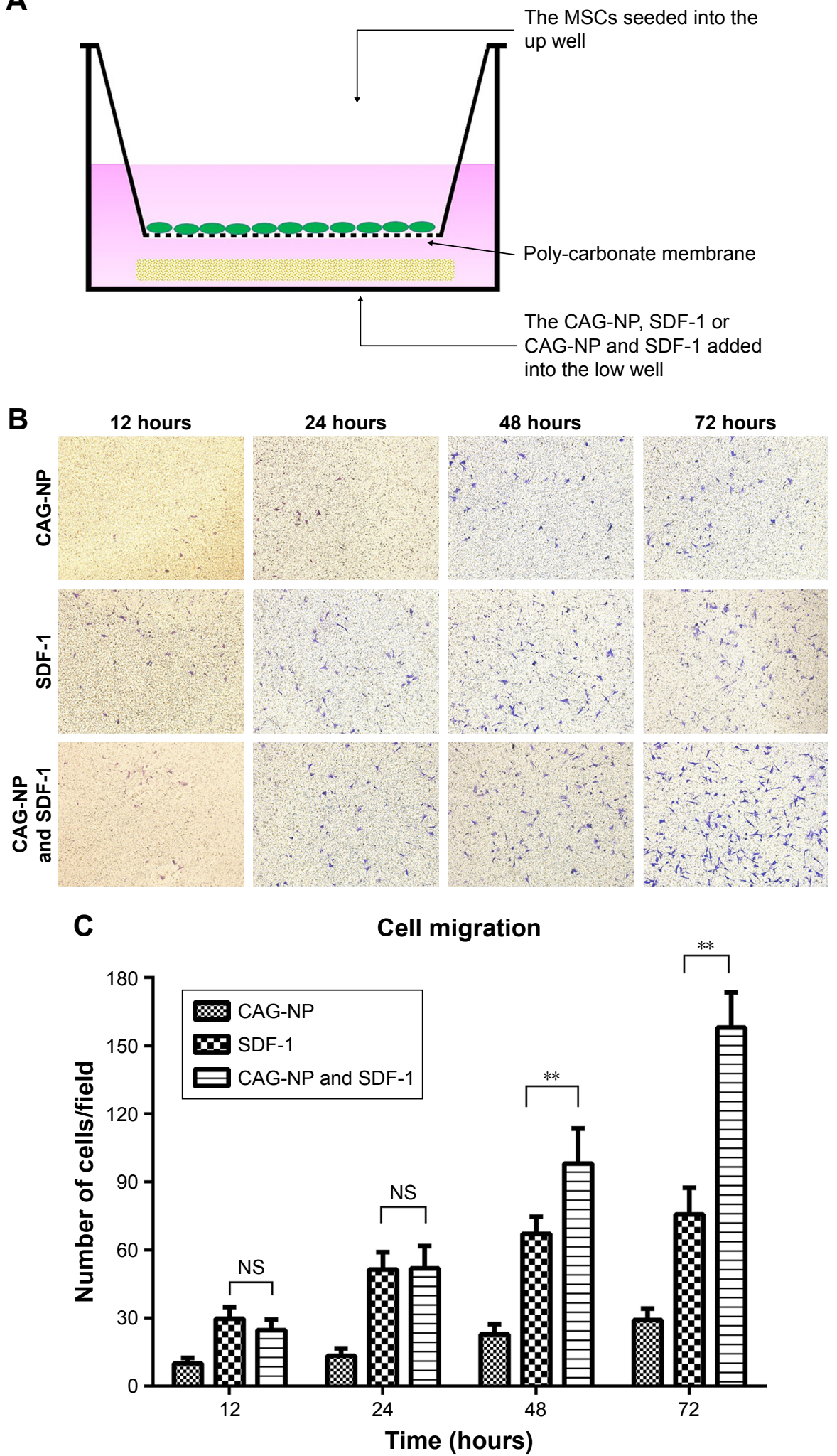

Figure 6 An in vitro Transwell migration model was used to analyze the ability of SDF-I released from NP-modified scaffolds to induce site-directed migration of stem cells.

Notes: (A) Pictorial images of transmigrated MSCs in response to SDF-I only, CAG-NP control, and NP-modified scaffolds with loaded SDF-I in a Transwell assay. (B) Representative images of transmigrated MSCs in response to the SDF-I only, CAG-NP control, and NP-modified scaffold with loaded SDF-I in a Transwell assay. Magnification is $100 \times$. (C) Average number of transmigrated MSCs in a Transwell migration assay after I2, 24, 48, and 72 hours of incubation. Results are mean values \pm SEM of five different fields from three independent experiments. $* * P<0.01, n=15$. Results are mean values \pm SD of five different fields from three independent experiments. $* \mathrm{P}<0.05, * * \mathrm{P}<0.01, \mathrm{n}=15$.

Abbreviations: CAG, chitosan-agarose-gelatin; MSCs, mesenchymal stem cells; NP, nanoparticle; NS, nonsignificant; SDF-I, stromal cell-derived factor I. 
A

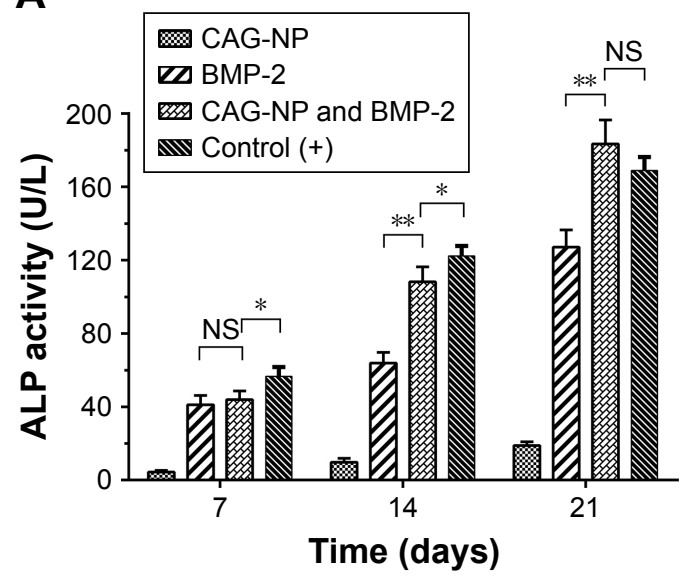

B
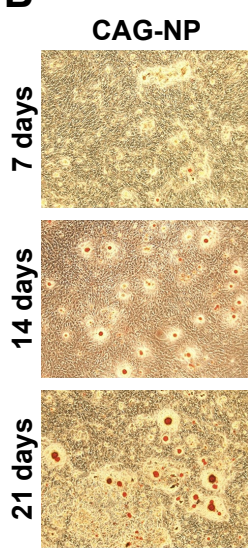

CAG-NP

and BMP-2
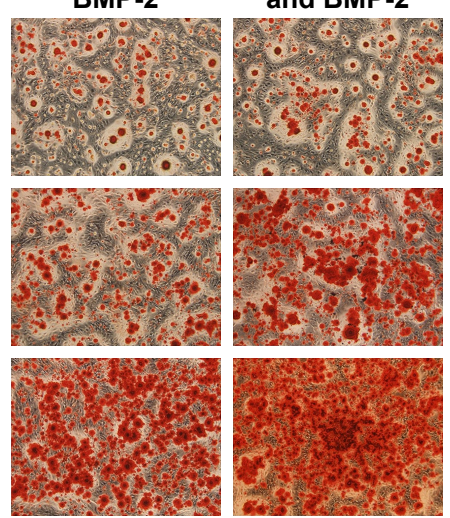

Control (+)

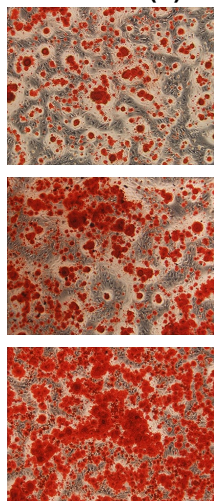

Figure 7 (A) Analysis of the ALP activity of MSCs seeded into the 24-well plates and cultured with the materials for up to 2I days. (B) Alizarin red staining of MSCs seeded on scaffolds for up to 21 days: the CAG-NP group was incubated with minimum medium and the positive control (+) group was incubated with osteoinductive medium. Magnification is $100 x$.

Notes: Results are mean values \pm SEM. $* \mathrm{P}<0.05, * * \mathrm{P}<0.0 \mathrm{I}, \mathrm{n}=4$ for each group.

Abbreviations: ALP, alkaline phosphatase activity; BMP-2, bone morphogenic protein-2; CAG, chitosan-agarose-gelatin; MSCs, mesenchymal stem cells; NP, nanoparticle; NS, nonsignificant; SEM, scanning electron microscopy.

biodegradable, noncytotoxic, nonimmunogenic, ${ }^{34,35}$ easy to process, absorbable, and having safe degradation end product. ${ }^{36,37}$ Our previous study about CSO/H NPs showed that they are stable in physiological $\mathrm{pH}$ solution and exhibited improved biocompatibility of chitosan-based nanoparticles. ${ }^{19}$ However, nanoparticles composed of CSO and heparin cannot easily meet the time stipulations of tissue engineering, which usually requires a period of a few weeks. In fact,
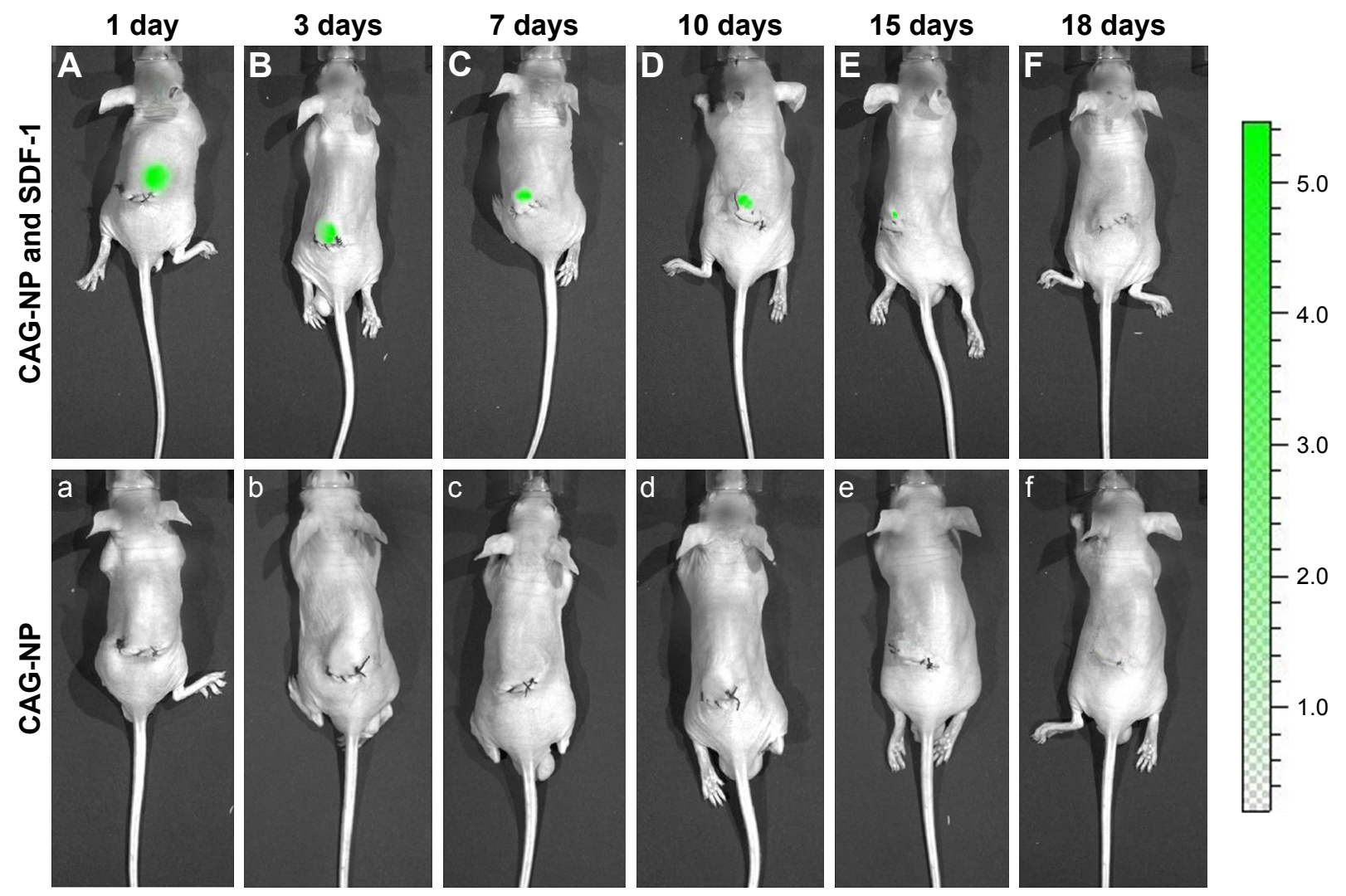

Figure 8 In vivo recruitment of MSCs by SDF-I released from NP-modified scaffold.

Note: The SDF-I released from the NPs-modified scaffold could recruit MSCs in vivo for more than 15 days, and the NPs-modified scaffold show no evident chemotactic activity, $n=3$. (A-F) CAG-NP \& SDF-I group, (a-f) CAG-NP group.

Abbreviations: CAG, chitosan-agarose-gelatin; MSCs, mesenchymal stem cells; NP, nanoparticle; SDF-I, stromal cell-derived factor I. 
as a cytokine delivery system, pure nanoparticles will be dispersed in a short time in vivo. ${ }^{19}$ In this study, the positively charged $\mathrm{CSO} / \mathrm{H}$ NPs were proven to modify the CAG scaffold by crosslinking to the negatively charged gelatin, and the CAG scaffold was used as a carrier for CSO/H NPs and protected the NPs from being dispersed by body fluid. Thus, the CSO/H NP-modified CAG scaffold could be used for sustained delivery of SDF-1 and BMP-2.

$\mathrm{CSO} / \mathrm{H}$ NPs were prepared by self-assembly. Like chitosan, CSO is suitable for developing self-assembled polymeric nanoparticles because of the availability for crosslinking with free amino groups and the cationic nature, which allows combination with multivalent anions. ${ }^{38}$ Heparin, with its multivalent anions and carboxylic group, can combine with CSO. The charge on the CSO/H NP is determined by the ratio of the surface molecules (CSO and heparin). The relatively larger absolute zeta potential value was associated with a comparatively small size and PDI. ${ }^{19}$ Therefore, we further optimized the ratio of CSO and heparin, and obtained nanoparticles with better size parameters (Table 1). The optimal size of nanoparticles designed for drug delivery is $\sim 50-150 \mathrm{~nm}$, which confers a high surface area-to-volume ratio. ${ }^{39}$ In this study, the particle size of the $\mathrm{CSO} / \mathrm{H}$ NPs varied from 92.4 to $100.7 \mathrm{~nm}$, suggesting an ideal size for drug delivery.

The three-dimensional microporous CAG scaffold was synthesized using a mixture of agarose and chitosan polymers with gelatin as a substrate for cells or CSO/H NPs. The polymers used could not individually satisfy all the desirable properties for tissue-engineered scaffolds. Agarose is an inert polymer, which does not favor cell adhesion but has good mechanical and elastic behavior; chitosan mimics the glycosaminoglycans structure, but its gels are brittle; and gelatin is very good for cell cultivation, but its gels have little mechanical integrity. So, we have used these polymers to align the properties required for tissue engineering and overcome their drawbacks via the synthesis of a blended scaffold. In the cryogelation process of the polymer reaction mixture, agarose self-gelates and chitosan were cross-linked by glutaraldehyde $(0.2 \%)$ in the presence of gelatin. Glutaraldehyde was used to cross-link the free amino groups present in chitosan and gelatin chains. ${ }^{24}$ There is another possibility of self-cross-linking of chitosan and gelatin chains. ${ }^{40}$ Different concentrations of polymers were utilized in this study (data not shown). Among the different combinations of varying polymer concentrations, we found good mechanical stability using a $1 \%$ chitosan concentration with $1 \%$ gelatin and $2 \%$ or $3 \%$ agarose in the polymer solution mixture (Table 2 ).
However, because of the greater number of negatively charged radicals exposed in gelatin, the CAG scaffold with $2 \%$ agarose could adsorb many more CSO/H NPs than the $3 \%$ agarose scaffold (Table 2, Figure 3 ).

The cytokines were loaded into materials after the NP-modified CAG scaffolds were prepared and not during their preparation. Preparation of most biomaterials involves organic solvents, heating, or electricity. ${ }^{41-43}$ Hence, cytokines may be inactivated if added during the preparation process. Therefore, the cytokines were not exposed to adverse conditions because they were loaded after the delivery system was prepared. The CSO/H NPs on the CAG scaffold can load not only a large amount of cytokines but also a variety of cytokines (including SDF-1 and BMP-2, Table 3), indicating that these nanoparticles could serve as a suitable carrier for cytokines. The hyaluronic acid hydrogels prepared by Holloway et al can also load 1.0 $\mu \mathrm{g}$ SDF-1 and BMP-2, ${ }^{22}$ and showed significant bone formation effect. Actually, the loading capacity of the scaffold is no more a problem because the physiologic of the cytokines concentration is much lower (1 $\mu \mathrm{g} / \mathrm{mL})$. Cytokines have a very short half-life and are very vulnerable to hostile environments. ${ }^{44,45}$ The SDF-1 and BMP-2 loaded in this material could maintain their bioactivity for more than 15 days, and the materials effectively achieved a sustained cytokine release profiles. Sustained release of cytokines allows for sustainable and effective bioactivity of the cytokines at the target position.

The golden standard for tissue engineering biomaterials does not involve the structure or the biochemical properties, but the favorable biocompatibility and the biological effect induced by the materials. We assessed the cellular compatibility of the CAG scaffold and the NP-modified scaffold with a CCK-8 assay. As shown in Figure 5B, the MSCs cocultured with the CAG scaffold and NP-modified scaffold had a growth curve similar to the MSCs in the control group. The MSCs seeded onto the NP-modified scaffold adhered to the scaffolds, and the cells exhibited a healthy morphology, demonstrating excellent cell compatibility of the NP-modified CAG scaffold.

Scaffolds, stem cells, and the active factors in the microenvironment are indispensable for tissue engineering. ${ }^{46,47}$ There are still no methods to meet all the requirements above, at present. In this study, we proposed a novel material for tissue engineering. The three-dimensional CAG scaffold with super micropores could enhance the MSC passage, waste transport, and nutrient supply efficiently throughout the scaffold. ${ }^{48,49}$ The in vitro MSC migration assay and the in vivo MSC recruitment study showed a strong chemotaxis ability of the NP-modified 
CAG scaffolds with loaded SDF-1. Therefore, we believe that NP-modified CAG scaffolds with loaded SDF-1 could recruit autologous MSCs in vivo for a long period of time. The in vitro osteogenesis study showed that the ALP activities induced by the scaffolds with loaded BMP-2 increased throughout the first 21 days, suggesting that the sustained release of BMP-2 could continually stimulate ALP expression. BMP-2 may be the most important active factor in the microenvironment for bone tissue engineering, ${ }^{50}$ and sustained release of BMP- 2 provides the scaffold with a much more favorable microenvironment. From the above, the CSO/H NP-modified CAG scaffold with loaded SDF-1 and BMP-2 will be a potential cytokine delivery scaffold for bone tissue engineering.

However, there are some limitations to our study. An animal model may need to be developed to investigate the availability and effectiveness of CSO/H NP-modified CAG scaffolds with loaded SDF-1 and BMP-2 for bone tissue repair and regeneration in vivo. Also, there are still several problems to be solved before the in vivo experiment. Firstly, the exposure time and concentration of SDF-1 can cause different biological effects, so we need to find a proper amount of SDF-1 we used in vivo, which make the SDF-1 exert the chemotactic functions but not promote primary tumor development, growth, and metastases. Secondly, we are trying to delay the release of BMP-2 after SDF-1 and the sequential release of cytokines may lead to a better synergistic effect. In addition, the structural strength of the scaffold is not sufficient, so further investigations are needed to refine these NP-modified CAG scaffolds so that they can completely meet the needs of bone tissue engineering or regeneration.

\section{Conclusion}

CSO/H NP-modified CAG scaffolds can be used as a cytokine delivery scaffold for tissue engineering or regeneration. CSO/H NPs can be prepared by self-assembly using CSO and heparin, and the CAG scaffolds can be synthesized with $\mathrm{CAG}$ and modified by CSO/H NPs. They have a excellent cell compatibility, high cytokine loading capacity, and can maintain the bioactivity of cytokines for more than 15 days. Therefore, the novel NP-modified scaffold may be a promising biomaterial for tissue engineering.

\section{Acknowledgments}

This work was supported by a grant from the Key Research and Development Program of Hunan Province (2018SK2103). The authors appreciate the editorial assistance from Editage Editing, People's Republic of China, in the preparation of this paper.

\section{Disclosure}

The authors report no conflicts of interest in this work.

\section{References}

1. Hokugo A, Saito T, Li A, Sato K, Tabata Y, Jarrahy R. Stimulation of bone regeneration following the controlled release of water-insoluble oxysterol from biodegradable hydrogel. Biomaterials. 2014;35(21): 5565-5571.

2. Lopez CD, Witek L, Torroni A, et al. The role of $3 \mathrm{D}$ printing in treating craniomaxillofacial congenital anomalies. Birth Defects Res. 2018;110(13):1055-1064.

3. Pensak M, Hong S, Dukas A, et al. The role of transduced bone marrow cells overexpressing BMP-2 in healing critical-sized defects in a mouse femur. Gene Ther. 2015;22(6):467-475.

4. Missori P, Morselli C, Domenicucci M. Transplantation of autologous cranioplasty in Europe as part of bone organ. Acta Neurochir. 2014 156(10):2015-2016.

5. Azi ML, Aprato A, Santi I, Kfuri M, Masse A, Joeris A. Autologous bone graft in the treatment of post-traumatic bone defects: a systematic review and meta-analysis. BMC Musculoskelet Disord. 2016;17(1):465.

6. Abolghasemian M, Sadeghi Naini M, Tangsataporn S, et al. Reconstruction of massive uncontained acetabular defects using allograft with cage or ring reinforcement: an assessment of the graft's ability to restore bone stock and its impact on the outcome of re-revision. Bone Joint J. 2014;96-B(3):319-324.

7. Wang B, Mei X, Liu W, Yu F. Chest wall reconstruction with 3-dimensional custom-made carbon fiber ribs. J Thorac Cardiovasc Surg. 2018;156(4):e177-e179.

8. Betz VM, Kochanek S, Rammelt S, Müller PE, Betz OB, Messmer C. Recent advances in gene-enhanced bone tissue engineering. $J$ Gene Med. 2018;20(6):e3018.

9. Hasan A, Byambaa B, Morshed M, et al. Advances in osteobiologic materials for bone substitutes. J Tissue Eng Regen Med. 2018;12(6): $1448-1468$.

10. Chapelier AR, Missana MC, Couturaud B, et al. Sternal resection and reconstruction for primary malignant tumors. Ann Thorac Surg. 2004, 77(3):1001-1007.

11. Arnold PG, Pairolero PC. Chest-wall reconstruction: an account of 500 consecutive patients. Plast Reconstr Surg. 1996;98(5):804-810.

12. Li H, Xue K, Kong N, Liu K, Chang J. Silicate bioceramics enhanced vascularization and osteogenesis through stimulating interactions between endothelia cells and bone marrow stromal cells. Biomaterials. 2014;35(12):3803-3818.

13. Amini AR, Laurencin CT, Nukavarapu SP. Bone tissue engineering: recent advances and challenges. Crit Rev Biomed Eng. 2012;40(5):363-408.

14. Alagesan S, Griffin MD. Autologous and allogeneic mesenchymal stem cells in organ transplantation: what do we know about their safety and efficacy? Curr Opin Organ Transplant. 2014;19(1):65-72.

15. Tang Q, Luo C, Lu B, et al. Thermosensitive chitosan-based hydrogels releasing stromal cell derived factor-1 alpha recruit MSC for corneal epithelium regeneration. Acta Biomater. 2017;61:101-113.

16. Li L, Zhou G, Wang Y, Yang G, Ding S, Zhou S. Controlled dual delivery of BMP-2 and dexamethasone by nanoparticle-embedded electrospun nanofibers for the efficient repair of critical-sized rat calvarial defect. Biomaterials. 2015;37:218-229.

17. Zhang H, Jia X, Han F, et al. Dual-delivery of VEGF and PDGF by double-layered electrospun membranes for blood vessel regeneration. Biomaterials. 2013;34:2202-2212.

18. Raftery RM, Mencía-Castaño I, Sperger S, et al. Delivery of the improved BMP-2-advanced plasmid DNA within a gene-activated scaffold accelerates mesenchymal stem cell osteogenesis and critical size defect repair. J Control Release. 2018;283:20-31.

19. Wang B, Tan L, Deng D, et al. Novel stable cytokine delivery system in physiological $\mathrm{pH}$ solution: chitosan oligosaccharide/heparin nanoparticles. Int J Nanomedicine. 2015;10:3417-3427. 
20. Xu X, Jha AK, Duncan RL, Jia X. Heparin-decorated, hyaluronic acid-based hydrogel particles for the controlled release of bone morphogenetic protein 2. Acta Biomater. 2011;7(8):3050-3059.

21. Ruppert R, Hoffmann E, Sebald W. Human bone morphogenetic protein 2 contains a heparin-binding site which modifies its biological activity. Eur J Biochem. 1996;237(1):295-302.

22. Holloway JL, Ma H, Rai R, Hankenson KD, Burdick JA. Synergistic effects of SDF-1 $\alpha$ and BMP-2 delivery from proteolytically degradable hyaluronic acid hydrogels for bone repair. Macromol Biosci. 2015;15(9): 1218-1223.

23. Hutmacher DW. Scaffolds in tissue engineering bone and cartilage. Biomaterials. 2000;21(24):2529-2543.

24. Bhat S, Tripathi A, Kumar A. Supermacroprous chitosan-agarosegelatin cryogels: in vitro characterization and in vivo assessment for cartilage tissue engineering. $J R$ Soc Interface. 2011;8(57):540-554.

25. Gupta A, Bhat S, Jagdale PR, et al. Evaluation of three-dimensional chitosan-agarose-gelatin cryogel scaffold for the repair of subchondral cartilage defects: an in vivo study in a rabbit model. Tissue Eng Part A.

26. Tan Q, Tang H, Hu J, et al. Controlled release of chitosan/heparin nanoparticle-delivered VEGF enhances regeneration of decellularized tissue-engineered scaffolds. Int J Nanomedicine. 2011;6:929-942.

27. Chiu LL, Radisic M. Scaffolds with covalently immobilized VEGF and angiopoietin-1 for vascularization of engineered tissues. Biomaterials. 2010;31(2):226-241.

28. Zhu H, Guo ZK, Jiang XX, et al. A protocol for isolation and culture of mesenchymal stem cells from mouse compact bone. Nat Protoc. 2010; 5(3):550-560.

29. Zhang H, Yu S, Zhao X, Mao Z, Gao C. Stromal cell-derived factor$1 \alpha$-encapsulated albumin/heparin nanoparticles for induced stem cell migration and intervertebral disc regeneration in vivo. Acta Biomater. 2018;72:217-227.

30. Hettiaratchi MH, Miller T, Temenoff JS, Guldberg RE, Mcdevitt TC. Heparin microparticle effects on presentation and bioactivity of bone morphogenetic protein-2. Biomaterials. 2014;35(25):7228-7238.

31. Xiong GM, Yap YZ, Choong C. Single-step synthesis of heparindoped polypyrrole nanoparticles for delivery of angiogenic factor. Nanomedicine. 2016;11(7):749-765.

32. Lee J, Yoo JJ, Atala A, Lee SJ. The effect of controlled release of PDGF-BB from heparin-conjugated electrospun PCL/gelatin scaffolds on cellular bioactivity and infiltration. Biomaterials. 2012;33(28):6709-6720.

33. Takikawa $\mathrm{M}$, Nakamura $\mathrm{S}$, Ishihara $\mathrm{M}$, et al. Improved angiogenesis and healing in crush syndrome by fibroblast growth factor-2-containing low-molecular-weight heparin (Fragmin)/protamine nanoparticles. J Surg Res. 2015;196(2):247-257.

34. Thomas AM, Gomez AJ, Palma JL, Yap WT, Shea LD. Heparin-chitosan nanoparticle functionalization of porous poly(ethylene glycol) hydrogels for localized lentivirus delivery of angiogenic factors. Biomaterials. 2014;35(30):8687-8693.

35. Venkatesan J, Anil S, Kim SK, Shim MS. Chitosan as a vehicle for growth factor delivery: Various preparations and their applications in bone tissue regeneration. Int J Biol Macromol. 2017;104(Pt B):1383-1397. 2014;20(23-24):3101-3111.

36. Islam N, Ferro V. Recent advances in chitosan-based nanoparticulate pulmonary drug delivery. Nanoscale. 2016;8(30):14341-14358.

37. Shevtsov M, Nikolaev B, Marchenko Y, et al. Targeting experimental orthotopic glioblastoma with chitosan-based superparamagnetic iron oxide nanoparticles (CS-DX-SPIONs). Int J Nanomedicine. 2018;13: 1471-1482.

38. Lin YH, Chung CK, Chen CT, Liang HF, Chen SC, Sung HW. Preparation of nanoparticles composed of chitosan/poly-gamma-glutamic acid and evaluation of their permeability through Caco-2 cells. Biomacromolecules. 2005;6(2):1104-1112.

39. Choi S, Kim S, Bae YJ, Park JW, Jung J. Size-dependent toxicity of silver nanoparticles to Glyptotendipes tokunagai. Environ Health Toxicol. 2015;30:e2015003.

40. Han F, Yang X, Zhao J, Zhao Y, Yuan X. Photocrosslinked layered gelatin-chitosan hydrogel with graded compositions for osteochondral defect repair. J Mater Sci Mater Med. 2015;26(4):160.

41. Wang H, Li X, Mao K, et al. Electrochemical immunosensor for $\alpha$-fetoprotein detection using ferroferric oxide and horseradish peroxidase as signal amplification labels. Anal Biochem. 2014;465:121-126.

42. Messiaen AS, Forier K, Nelis H, Braeckmans K, Coenye T. Transport of nanoparticles and tobramycin-loaded liposomes in Burkholderia cepacia complex biofilms. PLoS One. 2013;8(11):e79220.

43. Wang Y, Guo X, Pan R, et al. Electrodeposition of chitosan/gelatin/ nanosilver: a new method for constructing biopolymer/nanoparticle composite films with conductivity and antibacterial activity. Mater Sci Eng C Mater Biol Appl. 2015;53:222-228.

44. Seo BB, Choi H, Koh JT, Song SC. Sustained BMP-2 delivery and injectable bone regeneration using thermosensitive polymeric nanoparticle hydrogel bearing dual interactions with BMP-2.J Control Release. 2015;209:67-76.

45. Dalonneau F, Liu XQ, Sadir R, et al. The effect of delivering the chemokine SDF- $1 \alpha$ in a matrix-bound manner on myogenesis. Biomaterials. 2014;35(15):4525-4535.

46. Park HJ, Yu SJ, Yang K, et al. Paper-based bioactive scaffolds for stem cell-mediated bone tissue engineering. Biomaterials. 2014;35(37): 9811-9823.

47. Gao S, Zhao P, Lin C, et al. Differentiation of human adipose-derived stem cells into neuron-like cells which are compatible with photocurable threedimensional scaffolds. Tissue Eng Part A. 2014;20(7-8):1271-1284.

48. Xu H, Cai S, Xu L, Yang Y. Water-stable three-dimensional ultrafine fibrous scaffolds from keratin for cartilage tissue engineering. Langmuir. 2014;30(28):8461-8470.

49. Terzaki K, Kissamitaki M, Skarmoutsou A, et al. Pre-osteoblastic cell response on three-dimensional, organic-inorganic hybrid material scaffolds for bone tissue engineering. J Biomed Mater Res A. 2013;101(8): 2283-2294.

50. Rawadi G, Vayssière B, Dunn F, Baron R, Roman-Roman S. BMP-2 controls alkaline phosphatase expression and osteoblast mineralization by a Wnt autocrine loop. J Bone Miner Res. 2003;18(10):1842-1853.

International Journal of Nanomedicine

\section{Publish your work in this journal}

The International Journal of Nanomedicine is an international, peerreviewed journal focusing on the application of nanotechnology in diagnostics, therapeutics, and drug delivery systems throughout the biomedical field. This journal is indexed on PubMed Central, MedLine, CAS, SciSearch $®$, Current Contents ${ }^{\circledR} /$ Clinical Medicine, Submit your manuscript here: http://www.dovepress.com/international-journal-of-nanomedicine-journal

\section{Dovepress}

Journal Citation Reports/Science Edition, EMBase, Scopus and the Elsevier Bibliographic databases. The manuscript management system is completely online and includes a very quick and fair peer-review system, which is all easy to use. Visit http://www.dovepress.com/ testimonials.php to read real quotes from published authors. 